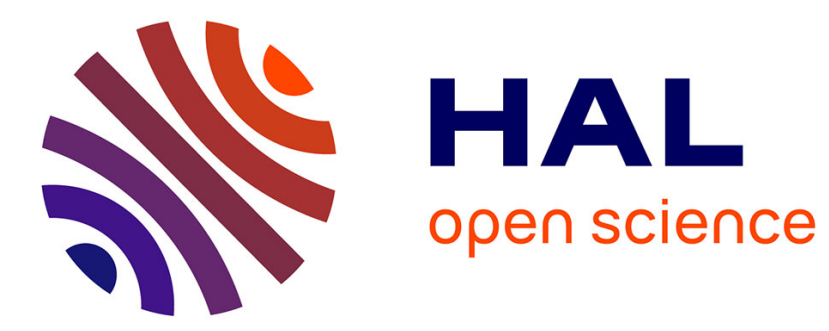

\title{
L'imaginaire découpé de Mon oncle de Jacques Tati
}

Marguerite Chabrol

\section{To cite this version:}

Marguerite Chabrol. L'imaginaire découpé de Mon oncle de Jacques Tati. Cahier Louis-Lumière, 2008, 5 (1), pp.25-31. 10.3406/cllum.2008.910 . hal-03248579

HAL Id: hal-03248579

https://hal-univ-paris8.archives-ouvertes.fr/hal-03248579

Submitted on 3 Jun 2021

HAL is a multi-disciplinary open access archive for the deposit and dissemination of scientific research documents, whether they are published or not. The documents may come from teaching and research institutions in France or abroad, or from public or private research centers.
L'archive ouverte pluridisciplinaire HAL, est destinée au dépôt et à la diffusion de documents scientifiques de niveau recherche, publiés ou non, émanant des établissements d'enseignement et de recherche français ou étrangers, des laboratoires publics ou privés. 


\section{L'imaginaire découpé de Mon oncle de Jacques Tati}

\section{Marguerite Chabrol}

\section{Résumé}

La notion de montage dans le plan ne suffit pas à rendre compte de la mise en scène de Tati, en particulier dans Mon oncle, car le réalisateur tend à travailler sur les séparations entre les différentes composantes de l'image plutôt que sur leurs liens. II confie ainsi au spectateur la responsabilité d'une forme de montage, d'associations d'idées qui font naître sous forme d'images mentales les thèmes fondamentaux du film.

\section{Abstract}

Jacques Tati has a very specific way of editing, especially in My Uncle. Instead of putting various elements together within the frame or through editing, he separates them. The spectator must virtually do the editing work by playing some sort of Memory game with scatterered elements, and thus discover the main themes of the movie.

\section{Citer ce document / Cite this document :}

Chabrol Marguerite. L'imaginaire découpé de Mon oncle de Jacques Tati. In: Cahier Louis-Lumière $n^{\circ} 5,2008$. Coupe, découpe, découpage. pp. 25-31;

doi : 10.3406/cllum.2008.910

http://www.persee.fr/doc/cllum_1763-4261_2008_num_5_1_910

Document généré le 18/01/2018 


\section{L'imaginaire découpé de Mon oncle de Jacques Tati}

\author{
| Marguerite Chabrol
}

\section{Résumé}

La notion de montage dans le plan ne suffit pas à rendre compte de la mise en scène de Tati, en particulier dans Mon oncle, car le réalisateur tend à travailler sur les séparations entre les différentes composantes de l'image plutôt que sur leurs liens. Il confie ainsi au spectateur la responsabilité d'une forme de montage, d'associations d'idées qui font naître sous forme d'images mentales les thèmes fondamentaux du film.

\section{Abstract \\ Jacques Tati has a very specific way of editing, especially in My Uncle. Instead of putting various elements together within the frame or through editing, he separates them. The spectator must virtually do the editing work by playing some sort of Memory game with scatterered elements, and thus discover the main themes of the movie.}

Connu pour explorer le plan en profondeur et dans la durée, Jacques Tati semble davantage avoir recours au montage dans le plan qu'au montage dans le sens traditionnel. Mon oncle est un des films où ce travail est le plus abouti. De nombreuses analyses de cette œuvre' ont montré que ce montage dans le plan repose autant sur des dissociations que sur un véritable assemblage.
Tati en effet décompose et découpe le gag, en s'appuyant sur des discontinuités, par exemple entre le son et l'image ou entre le premier et l'arrière-plan, entre la cause et l'effet, etc ${ }^{2}$. Mais on ne peut réduire la fragmentation chez Tati à sa manière de s'approprier le genre burlesque et d'en détourner certains clichés ou de le nourrir d'une forme de suspense toute personnelle. Derrière ce fonctionnement, les thématiques plus profondes du film sont elles aussi traitées selon une logique de découpage qui peut paraître paradoxale, dans la mesure où beaucoup des motifs essentiels du film ne sont finalement présents qu'en filigrane.

Nous nous proposons d'envisager la notion de découpage chez Tati - et dans Mon oncle en particulier, qui systématise ce procédé - non pas dans son sens traditionnel lié au montage, c'est-à-dire d'agencement de plans décomposant une action dans une recherche de continuités, mais comme une pratique de mise en scène plus générale consistant à fragmenter les images mentales que Tati cherche à faire naître chez le spectateur.

Le cinéaste découpe, sépare : il dissocie le son et l'image, il a recours à de légères ruptures dans le passage d'un plan à l'autre, afin de ne jamais proposer de raccord ou de coupe "transparente", et il découpe aussi ses thèmes récurrents en les disséminant au fil de son film. Au spectateur de monter. Malgré le rôle essentiel du cadrage et du plan fixe, les plans ne sont jamais entièrement autonomes, ils doivent être associés à d'autres par le spectateur à qui ils sont proposés de manière discontinue.
1

Dès le texte d'André Bazin, qui évoque la manière dont le cinéaste "détruit la netteté par la netteté", dans "Monsieur Hulot et le temps", Esprit, mai 1953, repris dans Qu'est-ce que le cinéma ?, Paris, Éd. du Cerf, 1975.
2

ce point, voir par exemple Francis RAMIREZ et Christian ROLOT, Mon Oncle. Jacques Tati, Paris, Nathan, coll. Synopsis, 1993, ou Stéphane Goudet, Jacques Tati, de Francois le facteur à Monsieur Hulot, Paris, Cahiers du cinéma / CNDP, coll. "Les Petits Cahiers", 2002.
3

Voir sur ce point la distinction entre montage et découpage proposée par Vincent Amiel dans Esthétique du montage, Nathan, 2001, p. 6-8. 


\section{- Le film comme jeu de construction}

Le spectateur de Mon oncle est sans cesse amené à reconstruire les éléments du film. Cela commence par les personnages qui, au-delà des stéréotypes à partir desquels ils sont composés, sont souvent découpés en un corps stylisé et une expressivité déplacée vers d'autres objets. Les émotions des personnages se manifestent ainsi souvent à travers les objets et non dans le jeu des acteurs. On rejoindra de ce point de vue les analyses de Vincent Amiel sur Buster Keaton*, qui montre par exemple comment, dans Le Mécano de la "General", la tristesse du héros est incarnée par les mouvements des roues de la locomotive. La dissociation du corps et de la psychologie du personnage provoque ainsi un effet poétique qui s'épanouit particulièrement dans le genre burlesque. Dans Mon oncle, Tati prête ainsi des émotions fortes à des personnages froids dans leur comportement : la colère de $M$. Arpel face au solex de Hulot qui occupe sa place de parking à l'usine, ou lors de sa découverte des arbres égalisés, est ainsi exprimée par deux plans subjectifs pris depuis la voiture, desquels le corps du personnage est absent. Le point de vue est alors celui de la voiture elle-même, plutôt que celui de son conducteur, dans la mesure où le cadre est très centré par rapport au pare-brise et la caméra semble placée plus en avant que sur le siège du conducteur. Chez Hulot, ce sont plutôt les fonctions physiologiques qui sont déléguées aux machines : lorsqu'il s'endort à l'usine, sa respiration est prise en charge par le tuyau qui jette de la vapeur en rythme; puis sa faim est évoquée par les saucisses. Dans ces deux exemples, le personnage déborde son propre corps et doit être reconstruit par le spectateur. Le spectateur est plus généralement invité à appréhender le déroulement du film comme un jeu de memory, c'est-à-dire à fixer dans sa mémoire certaines images qui seront évoquées à nouveau par la suite. Les gags effilochés, les répétitions peuvent se comprendre dans cette perspective de réactivation de la mémoire du spectateur. Le poisson-jet d'eau forme une paire avec le poisson du cabas de Hulot; les fauteuils bas des Arpel en forment une avec ceux de l'usine ; la charrette qui passe régulièrement fait aussi jouer la mémoire du spectateur; il en va de même de la série des hublots (l'usine, l'œil de bœuf de la maison, la porte du garage). Chaque répétition, à cause de légères variations par rapport à la précédente occurrence de l'objet, est avant tout une réminiscence. Des liens sont ainsi à construire entre les scènes, et c'est au spectateur de reconnaître par exemple la force du lien entre Hulot et Gérard, au fait qu'ils jouent tous les deux, volontairement ou non, avec les nénuphars qui décorent le bassin. Tati reprend aussi de cette façon certains topoi burlesques. Le célèbre gag de l'arroseur arrosé est ainsi traité dans Mon oncle en deux vignettes appartenant à deux scènes distinctes : celle du marchand mouillé par Hulot, puis celle où Hulot lui-même se retrouve les pieds dans le bassin des Arpel. La zone du pantalon de Hulot qui se trouve dans l'eau correspond exactement à la partie du pantalon du marchand qu'il avait éclaboussée. Le spectateur averti pourra éventuellement saisir l'unité de tels gags ou d'images essentielles du film, s'il procède à la confrontation que le montage du film refuse de faire. 
D’autres séquences s'organisent plus en profondeur autour d'associations d'idées, plutôt qu'autour d'unités d'action : l'intrigue est toujours présentée comme anecdotique, routinière ou dérisoire. Il faut souvent mettre à jour dans les séquences un élément d'unité plus ou moins apparent : ainsi, le thème du temps surgit discrètement dans de nombreuses séquences, découpé en différents éléments comme un bruit de battement, la présence d'un sablier, quelques répliques, etc., comme le montrent les analyses de Stéphane Goudet5. On ajoutera que la scène au cours de laquelle Gérard casse les arbres du jardin de ses parents évoque la radio, par la forme des arbres qui imitent des antennes et un grésillement métallique à l'arrière-plan, ce qui incarne très précisément les difficultés de communication que ressent le garçon. De la même manière, après que Mme Arpel a désigné Hulot comme le "cavalier" de la voisine lors de la garden party, le rival arrive précisément dans une tenue adéquate, et est présenté comme "un cavalier extraordinaire", ce qui contribue à ridiculiser ceux qui prennent les expressions au pied de la lettre.

\section{L'eau, les flux}

Plusieurs des exemples déjà évoqués le montrent déjà, les motifs découpés et répartis dans le film empruntent beaucoup à l'imaginaire de l'eau. Ce motif, important sans être très ostensible, nous intéresse ici non pour ses symboliques, quoique celles-ci puissent en partie fonctionner, mais pour la manière dont il est travaillé. Entre le jet d'eau des Arpel et le corps de tuyaux jeté dans la rivière, en passant par divers jeux d'enfants, une garden party aquatique, ou le parapluie inutile de Hulot, l'eau apparait pourtant sous les formes les plus diverses : dans le décor ou l'action de multiples séquences ou à travers des objets qui lui sont liés (contenants, protection contre l'eau...). Elle trouve en particulier une forte présence dans la bande sonore du film où les divers bruits qu'elle peut produire sont amplifiés. Derrière son rôle burlesque, ou sa manière de traduire l'opposition centrale du film entre monde ancien et monde moderne, l'eau est un motif décomposé en de multiples détails que le spectateur doit une nouvelle fois rassembler entre eux pour faire émerger des lignes de force. Elle organise en profondeur la structure imaginaire du film, liant entre eux certains personnages, demandant au spectateur de construire ces liens sans les lui imposer comme une évidence.

Les fluides servent ainsi à suggérer un principe de continuité au sein de l'imagerie très découpée que propose le réalisateur.

Le premier aspect de cette opposition entre les images découpées et l'imaginaire liquide rejoint la satire à laquelle se livre Tati dans Mon oncle. L'eau sert ainsi fondamentalement à organiser l'opposition entre contrainte et liberté qui structure le film : les images découpées sont du côté du monde moderne, décousu, stérile, tandis que les fluides deviennent le principal outil d'évasion ou de résistance des personnages positifs. La séquence des tuyaux à l'usine, construite fondamentalement autour de cette idée de débordement et d'évasion de Hulot et des ouvriers, s'appuie ainsi sur la présence de l'eau : la communauté ouvrière vivante

Voir Stéphane Goudet, "Tout communique", en bonus du coffret DVD Mon oncle, Naïve, 2005. 
- anime les arrière-plans, d'abord par ce jet d'eau et ensuite par le passage des ouvriers portant des tuyaux. Le monde vivant des ouvriers est ainsi caractérisé par l'eau et plus tard, les ronflements, la faim...

L'eau représente ainsi un dynamisme à l'intérieur d'un monde figé. Elle est par excellence ce qui anime l'image fixe : à l'intérieur de cadrages immobiles et relativement longs, Tati introduit en effet souvent un mouvement ou une animation par l'eau. Les deux exemples les plus frappants sont sans doute liés à la représentation du travail : le jeu de Gérard avec la baleine de son livre de sciences naturelles et le sommeil de Hulot à l'usine. Le livre de sciences naturelles comporte ainsi en couverture une image dessinée, figée, à laquelle l'eau donne vie. On peut ajouter que Gérard a déjà recours à un petit tuyau, ce qui est prémonitoire du morceau de bravoure du film et construit ainsi un autre lien entre les personnages, plus ténu mais non moins solide que les farces et promenades en solex qu'ils partagent. La scène est filmée en deux plans, un simple champ/contrechamp. Mais cette figure de montage n'a pas sa vocation habituelle de lien ou de réunion des deux espaces qui se font face. Au contraire, le champ/contrechamp s'organise autour d'une rupture, puisque les Arpel commettent une erreur d'interprétation. La coupe entre les deux plans a ici une valeur très forte.

Ouant à Hulot, il semble que la vapeur qui s'échappe du robinet gouttant au premier plan, se substitue à ses propres ronflements.

Dans ces deux exemples, fondés sur une vision très enfantine du travail, la tâche à accomplir apparaît comme rébarbative, figée, tandis que l'eau permet une évasion : non pas une évasion réelle, puisque le personnage reste prisonnier d'un lieu réduit bien souligné par le cadrage. Mais une évasion dans le domaine imaginaire.

C'est précisément en sollicitant l'imagination que l'eau remplit sa deuxième fonction, la plus essentielle dans le film, consistant à donner véritablement à Mon oncle sa tonalité mélancolique.

Si l'eau permet à Hulot ou Gérard de résister au modèle imposé par les Arpel, c'est aussi, comme nous l'avons évoqué, sur le mode d'un repli vers l'imaginaire. L'eau est fondamentalement l'instrument d'une libération individuelle, qui ne passe pas toujours par un rattachement à la communauté : le jeu de Gérard avec le bassin a une tonalité plus sombre que le jeu des enfants dans le caniveau. Ce passage suit immédiatement le moment où l'enfant a découvert l'absence de sa mère, remplacée par l'aspirateur qui fonctionnait tout seul, et où il est particulièrement pensif. L'eau se présente alors comme un miroir. Quant aux rêves de Hulot à l'usine, s'ils permettent d'échapper à un travail mécanique, ils nous restent de toute façon inaccessibles. Et l'image du miroir y intervient aussi, puisque la flaque au sol reflète une partie du décor. Au spectateur une nouvelle fois de monter ensemble ces deux plans pour percevoir une insistance du metteur en scène. En plus de l'animation qu'elle apporte, l'eau sert aussi à donner une "profondeur" à l'image, pourrait-on dire en reprenant un terme bachelardien. Ces amorces très discrètes d'introspection n'aboutissent pas dans Mon oncle. L'idée d'une intériorité ou d'un inconscient affleure sous forme de questions : à quoi rêve Hulot ? Ou'est-ce que Gérard attend de ses parents ? Ajoutons que l'accessoire le plus caractéristique de Hulot est son parapluie. Comme le souligne David Bellos ${ }^{6}$, c'est
6

David BELLOS, Jacques Tati, sa vie et son art, trad. de Pascale Voilley, Paris, Le Seuil, 2002. Ce passage se trouve p. 210 de l'édition anglaise, Jacques Tati : His Life And Art, Londres, Harvill press, 1999. 
un instrument de protection

d'autant plus inutile qu'il ne pleut

jamais chez Tati. Bellos y voit

l'emblème du caractère démodé

de Hulot et de son appartenance à un autre temps. On peut ajouter qu'on voit particulièrement le parapluie lorsque Hulot est en situation de dialogue difficile (par exemple avec sa jeune voisine, ou lorsqu'il refuse l'invitation de $M^{\text {me }}$ Arpel, par un geste négatif du parapluie). Le parapluie apparaît ainsi comme un obstacle à l'échange : l'objet apparaît aussi lorsque Gérard "échappe" parfois à son oncle pour aller jouer avec les autres enfants. Cet objet lié à l'eau et replié en permanence caractérise ainsi le personnage par une fermeture et une protection constantes qui contribuent aussi à son isolement. Imaginaire, repli sur soi sont ainsi évoqués de manière épisodique, par des objets ou des situations appartenant à un même univers thématique.

Il en va de même avec les images animales et aquatiques qui permettent à Tati un jeu avec les clichés assez développé. Les différentes métaphores animales et aquatiques qui jalonnent le film comportent une double dimension satirique et plus sombre. Tati utilise ainsi certains clichés en leur donnant une nouvelle tonalité : le poisson du cabas de Hulot et celui du jardin évoquent irrésistiblement l'image d'un "poisson hors de l'eau" et, derrière la personnification burlesque, suggèrent aussi un léger malaise (par la réaction violente du chien, ou par l'évocation d'une sorte d'agonie du poisson-jet d'eau). Truffaut n'était guère enthousiasmé par ce poisson' : il contribue pourtant à la création d'images très cohérentes. Dans le monde ancien, comme dans le monde moderne, il existe, de manière plus ou moins nette, une sensation d'étouffement, portée par le conflit entre l'air et l'eau. Lorsque le poisson s'anime et semble suffoquer devant le

livreur, la réaction effarée de ce dernier contribue à la dimension burlesque de la scène en en faisant un relais du spectateur. Mais la sensation physique portée par le son est très forte. Comme le note Michel Chion, "un poisson, c'est ce qui passe, c'est là sous l'eau, ça jaillit, ça disparaît, vif comme l'inconscient."8 Or, dans Mon oncle, les poissons manquent de vivacité et d'énergie. L'eau porte ainsi, à travers cette figure du poisson, une des grandes tensions qui organisent le film, celle entre l'inertie et le mouvement.

Cet arrière-plan imaginaire intervient aussi à travers les multiples expressions évoquées littéralement par certaines scènes du film (ajoutons le fait que la fête "tombe à l'eau" ou que le jet d'eau perd sa clarté d'“eau de roche”, etc.). Ce sont des expressions langagières que la mise en scène convoque, sans les utiliser explicitement. Chaque occurrence de l'eau dans le film évoque une nouvelle expression à qui Tati rend toute sa force visuelle, tandis que le langage à proprement parler est représenté dans les dialogues comme entièrement vide de sens. Le réalisateur coupe ici le signifiant du signifié, donnant au second une nouvelle forme. Cette séparation est d'ailleurs très concrètement évoquée dans Mon oncle par la manière dont Tati met en relation contenants et contenus. Les adultes privilégient les contenants, tandis que Hulot et les enfants sont des personnages libérant les contenus de leurs enveloppes. Notons que ce qui déborde de l'usine et du monde moderne sont des tuyaux, c'est-à-dire des contenants vides. Comme dans la garden party, ce n'est pas l'eau qui
7

Francois Truffaut, "Mon Oncle", Arts, 1958, repris in Les Films de ma vie, Paris, Flammarion, 1975 , p. 257-259.
8

Michel Chion, Jacques Tati, Paris, Cahiers du Cinéma, coll. Auteurs, 1987, p. 100 
- déborde de manière démesurée. La modernité apparaît ainsi comme un monde de contenants, de tuyaux, d'habillage (la voiture rutilante, les costumes tape-à-l'œil) ; alors que chez Hulot, l'importance va surtout aux contenus : lorsqu'il explore la cuisine des Arpel par exemple, il détourne les contenants vides de leur usage (il fait rebondir la carafe) ou les casse (le verre). Lorsque la carafe d'orangeade - boisson favorite des Arpel qui ne boivent jamais d'eau - remplit le verre toute seule à cause des diverses inclinaisons de la table, seul Hulot semble percevoir ce miracle, pourtant souligné par un bruitage très marqué. Le liquide sert ainsi à représenter un événement particulier, qui échappe à la monotonie caractéristique de la vie des Arpel et ceux-ci ne le voient même pas. Les dissociations auxquelles se livre le réalisateur vont ainsi jusqu'à systématiquement séparer ce qu'on pourrait résumer rapidement par le fond et la forme.

La dramatisation des clichés liés à l'eau réapparaît dans la scène du faux cadavre jeté à l'eau, qui repose sur la même articulation du burlesque et du dramatique. Tati convoque plusieurs images traditionnellement associées aux bords de l'eau la nuit : le cliché sentimental (qui permettra le quiproquo burlesque) et le cliché dramatique (la tentative de suicide). Si la scène se dénoue de façon comique et festive, le passage autour du pont est légèrement différent : le cadrage est moins composé que dans le reste du film, le découpage - au sens traditionnel cette fois - plus serré et les mouvements de caméra plus nombreux, ce qui rend le style plus réaliste. La scène est aussi construite selon un axe vertical et non dans la perspective horizontale qui domine dans le reste du film. Ce traitement donne d'autant plus d'importance à l'eau en tant qu'élément naturel, bien qu'on la voie assez brièvement. D'une part, on notera que cette rivière ne semble conduire nulle part. L'obscurité est particulièrement dense sous le pont et l'eau ne permet pas d'échapper aux espaces récurrents du film. La course-poursuite se déroule ensuite dans des lieux déjà familiers et les restes du mannequin de tuyaux demeurent en partie dans la charrette. Cela renforce l'idée d'épuisement (devant la difficulté à se débarrasser des tuyaux) et d'impossibilité à échapper à la dialectique entre monde ancien et monde moderne, puisque ce troisième espace est envahi par leur conflit.

D'autre part, par son style alors plus réaliste, Tati invite à prendre au sérieux les clichés évoqués dans cette scène. L'interruption de la scène sentimentale, déjà amorcée dans la séquence précédente, rappelle aussi l'absence ou la difficulté d'expression de sentiments dans le monde de Tati, qu'il soit ancien ou moderne. L'eau est donc le moyen indirect d'introduire des questionnements ou des tensions dans un film qui en est en apparence dépourvu. Il permet de renforcer sa tonalité mélancolique, ce qui peut souligner la dimension nostalgique et la perception du temps proposée par Mon oncle.

\section{L'image découpée}

Quoiqu'éparpillée sous des formes et dans des scènes diverses, l'eau nous semble être un des motifs structurant le plus fortement Mon oncle, mais il en existe probablement d'autres à explorer. On ne peut qu'insister sur la richesse des images chez Tati. Par image, nous entendons ici un 
arrière-plan imaginaire actualisé ponctuellement par différents détails visuels ou sonores. Ce motif présent à l'arrière-plan n'est en tout cas jamais représenté directement, il est appréhendé sur le mode de l'évocation. Finalement, Tati semble privilégier l'image au sens poétique du terme à l'image plus littérale du cinéma. Là où Nicole Brenez évoque pour d'autres films la notion d'invention figurative', on peut préférer pour Tati et Mon oncle l'idée du découpage, qui rend mieux compte d'un parti-pris de mise en scène fondé essentiellement sur la dissociation. En outre, Tati ne travaille pas particulièrement l'élaboration de figures dans Mon oncle, il tend à ne rien représenter de précis à travers ces images d'arrière-plan. Et il sépare plutôt qu'il ne cherche à construire, c'est aussi ce que suggèrent les scènes de chantier du film, qui au début évoquent des travaux de construction, mais à la fin sont plutôt une opération de destruction. Le spectateur reste finalement dépositaire des fonctions d'assemblage ou de montage.

\section{MARGUERITE CHABROL}

Marguerite Chabrol est maître de conférences en études cinématographiques à l'Université Paris $X$ - Nanterre. Elle travaille sur des questions d'esthétique, notamment sur le classicisme hollywoodien et les relations du cinéma avec les autres formes d'art (théâtre, musique). Elle est notamment l'auteur d'une thèse sur "La théâtralité dans le cinéma classique hollywoodien : les mises en scène de George Cukor et Joseph L. Mankiewicz" et co-auteur de Pickpocket de Robert Bresson (Atlande, 2005). 